\title{
A review of social collaborative network research.
}

\author{
WangYan ${ }^{1}$ a * \\ ${ }^{1}$ Dalian University of Science And Technology, Dalian, \\ 2 Dongbei University of Finance and Economics, 116052
}

Key words: cooperative network, social dynamic mechanism

\begin{abstract}
In recent decades, with the development of Internet technology, collaborative networks have emerged in a large number and presented a stronger trend of socialization.Focus of the "wisdom of crowds" collaborative network, effective implementation of the enterprise innovation, improve the innovative ability of enterprise, regional and even national, and promote economic development and social progress also has significant practical significance.This article from the concept of social collaboration network, the dynamic model of the social collaboration network and social influence on social writing network of existing research are reviewed, this to promote domestic related theory research and business practice have reference value.
\end{abstract}

\section{The introduction}

The collaborative activities among human groups are gradually formed with the emergence of human society. The members of society work together to achieve the goals that are difficult to accomplish alone. Due to the limitation of time and space, the early collaborative activities were limited to a small scope. However, with the continuous progress of communication technology, the scale of social individuals involved in collaborative activities has been increasing and the trend of networking has gradually emerged ${ }^{[1-5]}$, covering many social fields such as business and science, such as enterprise innovation network ${ }^{[6]}$ Network of scientists ${ }^{[7]}$.The success of these social collaboration network also suggests that the focus of the "wisdom of crowds" collaborative network to solve the complicated problem of ability and good application prospect, for social collaboration network further study can not only understand social collaboration network evolution mechanism and the internal dynamic mechanism, and effective implementation of the enterprise innovation, improve enterprise, regional and even national innovation ability, thus promoting economic development and social progress also has significant practical significance.

\section{The concept of social collaboration networks}

Social collaboration network is mainly composed of a number of social individuals, corporations, organizations or individuals) loosely gathered in the form of self organization, the social individual through spontaneous coordination and cooperation between each other, to find and solve problems, so as to achieve individual cannot be reached or need to pay high cost of individual goals $^{[8]}$.Members of the characteristics of this kind of collaborative network is a network of the whole target does not have a complete planning, but is entirely through spontaneous coordination and provide interaction with other members in the network, in the case of lack of central control, the spontaneity of the members of a network has a strong, corresponding relationship between members also has a strong variability.However interaction relationship between the main body of spontaneity and variability is not completely random, often and social attribute of the related subject, prompting attributes of the differences between subject when choosing interacting objects show different behaviors $^{[18]}$, Centola (2011) through the online test about the relationship between individual similarity and interaction analysis are presented shows that social interactions between individuals, such as the characteristics of the "birds of a feather flock together", this kind of interaction inclination inevitably leads to the change of the social individual relations which in turn leads to the evolution of the collaborative network structure ${ }^{[10]}$. At the same time, interaction and interaction between subjects are always within a certain range, and circles of different relationship structures 
have different degrees of influence on subject attributes ${ }^{[11]}$ Therefore, the change of network structure will lead to the change of the subject attribute, which will lead to the change of its interactive relationship.

\section{Related research work of social collaboration network}

Since the rise of complex network theory at the end of last century, the academic circle has carried out extensive research on the complex network in the real world.Among the many existing researches, the related researches on complex adaptive networks mainly include network topology evolution and network dynamics.

The first line will be understood as a dynamic adaptive network system, the related research mainly focus on the evolution of the network structure over time in the present state of different, the two results are significantly Watts and Strogatze (1998) use of random edge reconnection mechanism in the process of rule to the random network evolution observed "small world" network;Barabasi and Albert (1999) obtained the scale-free characteristics of the network through the analysis of the co-authored network of research partners

The second main line focuses on the dynamics of related activities on complex adaptive networks, including opinion dynamics, cultural dynamics, and behavioral diffusion ${ }^{[12]}$. Opinion dynamics focuses on the change process of the state of opinion in the crowd. There are many achievements in this field.The majority principle model.Sznajd model ${ }^{[13]}$, the marginal trust model. Cultural dynamics and kinetics of similar opinions ${ }^{[14]}$, but in the study of cultural dynamics that cultural change compared with the change of opinion more fuzziness, the boundary between different cultures is not clear, Axelrod cultural diffusion model is put forward ${ }^{[15]}$. Culture of different groups in the change of state of the probe has caused social science, statistical physics, and other fields into the dynamics of culture, such as Centola (2007) of cultural drift (culture drift) for further discusses the influence of the formation. The diffusion of behaviors is mainly to analyze the adoption and dissemination of individual behaviors in specific network structures.

In recent years, the coevolution of network topology and network related dynamics has been discussed.Holme and Newman(2006) combined the exchange of opinions and the interaction between similar individuals to investigate the common evolution of opinions and network structure.Kimura and Hayakawa(2008) then added heterophily mechanism on the basis of Holme and Newman models for further study, and concluded that the network structure would present a more complex state and include a small-world structure ${ }^{[16]}$.Gracia-lazaro et al. (2011) proposed the tolerance of social individuals to cultural differences on the basis of Axelrod model, and investigated the co-evolution of cultural groups and network structures under the network environment ${ }^{[17]}$.In conclusion, social interaction between the individual behavior in real collaborative network evolution and network topology evolution is a process of mutual influence and mutual evolution between, so for collaborative network dynamics mechanism of the further study need to be the change of individual behavior, as well as the evolution of the network structure, so as to further understand the dynamics of this common evolution process.

\section{Research on social dynamic mechanism based on individual interaction}

Research on social dynamic mechanism based on homophily

Lazarsfeld and Merton (1954) ${ }^{[12]}$ Homophily is proposed, homo stands for similarity, phily stands for like, and homophily describes social phenomena in which individuals tend to interact with individuals who are similar to them.

Research on homophily has two notable features. One of the characteristics is that the research background has changed. Due to the traceability of some network behaviors, many studies have begun to further explore the previous studies against this online background. Another characteristic is that the subject of research is no longer limited to human beings, and the academic circle gradually matches the similarity of attributes between other social individuals with the phenomenon of "birds of a feather flock together". 
Ruef et al. (2003) between the homophily as entrepreneurs officially one of the mechanisms for the formation of social groups, study relative to the identity factors lead to the change of individual choice behavior, local similarity is more likely to lead to social division.Leicht (2005) proposed a method to measure the similarity between individuals and examined the social network structure under age similarity while verifying the method through simulation experiments.Kimura (2008) was investigated by using the method of ABM social individual views on the homogeneity and heterogeneity of tend to the relationship between the network structure change, and it is concluded that when the social individual opinion to maintain the homogeneity and heterogeneity, the social network will present a small world structure. The model is essentially HN (Holme \& Newman 2006) the expansion of the model, in the $\mathrm{HN}$ model joined the behavior selection mechanism of heterogeneous tendency, examines the homophily and heterophily effect on social individual at the same time, the network structure of state;Similar studies have been conducted by Bramoulle and Rogers(2009) on the properties and structures of homhphily in social networks.

Studies on the social dynamic mechanism based on heterophily

Although the relationship between social individuals showed a strong similarity, but there is a difference between the empirical fact that individuals in the social relationship exists in the social network, Page said: when the social individual needs to solve some social problems often need recognition and diversity of the problem, such as moon, cure of disease of new product design, etc.Heterogeneous social relations also exist in some social networks that lack central control. There are rich researches on diverse social relations and cooperative networks.

Heterophily describes social individuals in choosing a partner often tend to choose complements their own capabilities and technology of other social individuals, and then to solve some specific problems, corresponding the social relations show the diversity of individuals, related research is aimed at the team level.Moody (2004) concluded in the analysis of more than 30 years of research cooperation data that when research problems involved quantitative work in the management field, the cooperative relationship of the team tended to be diversified. Some of the more theoretical areas, diversity of cooperation less ${ }^{[2]}$.Jones et al. (2008) also found in the study of the composition of scientific research cooperation team gradually broke through the limit between university, and the formation of a high-level university in cooperation team diversity, and influential play an important role in a paper published. Many studies have carried out more in-depth discussions on this kind of task-based diversity relationship and task completion.Lu and Page (1998) proposed a model of collaborative problem solving, and concluded that the collective performance of diverse teams in solving complex problems was higher than that of elite teams and random teams.Knight (1999) analyzed the relationship between the cognitive diversity of the company's senior management team and the company's performance.Horwitz (2007) pointed out in his research that the performance of task-based diverse teams was higher than that of teams with only diverse social attributes. The diversity of team members can produce better performance and also appears in the team of inventors.Innovation and the efficiency of information use. In addition, about the related study also for the diversity of enterprise alliance cooperative relations to a certain extent, in the study of related social individual organizations or enterprises, corresponding relationship is the collaboration between enterprises. In a word, the generation of diversity relationship needs to be guided by certain task objectives and exists in the process of information transmission, decision-making and other similar processes.

And he thinks about homophily and heterophily

Through the analysis of the existing relevant research work, we can understand the interaction behavior of similar and diversified tendencies among social individuals as the following aspects.(1) in a free interactive environment, because the individual interaction has a lot of autonomy, the individual from the potential in the interactive object to select it shows some aspect of the social attributes, such as research interests with their close to other individuals, thus interaction has a strong tendency to attribute similarity.(2) when some interactive activities is to have a specific task goal orientation, the individual will evaluate their own can not complete the subtasks, and then select to complete these subtasks individuals interact, therefore the corresponding social relations 
show diversity. (3) in most studies on homophily and heterophily, the two behaviors are considered separately. In fact, there is some interaction between them in the process of social interaction. When individuals have the tendency of similarity interaction, the pursuit of similarity is not unlimited. At the same time, the diversity relationship between individuals does not mean that the relationship between any two individuals is completely heterogeneous, which must be similar in some aspect of social attributes. Therefore, it is necessary to consider the degree of different tendencies of the two behaviors in the subsequent studies, or to combine the two mechanisms organically for further exploration.

\section{Research on social impact}

Early psychoanalysis of social influences, such as Milgram's (1974) submission authority test, was carried out, Asch (1956) line judgment test, etc., subsequent studies included social cognition, and further discussed the role of social influence on individual behavior and decision-making.

Social influence on the social nature of the social relationship between the structure and the individual, so the change of social structure is also inevitably has the influence to the behavior of the social individual, related research mainly involves innovation, knowledge communication and individual attributes and behaviors. In the research on innovation activities, Burt (1987) earlier analyzed the adoption of innovation through structural similarity. Then Cowan et al.'s similarity to the organizational structure, dense clustering, hole structure,structural embeddedness. The factors of network structure such as the diffusion of innovation ideas and the emergence of innovation cooperation have been discussed. Innovation activities are also a process of knowledge exchange. Reagans and McEvil investigated the effects of cohesion and edge structure on knowledge communication activities. Lambiotte and Panzarasa explored the relationship between community structure and knowledge creation and information diffusion. The literature explores the diffusion of knowledge under the overall network structure. Studies on individual attributes and behaviors focus on the interaction and diffusion of individual attributes and social behaviors on the Internet.Fowler and Christakis et al. studied the spread of alcoholism, obesity, happiness and loneliness over large social networks.Judd and others under different network structure of coloring and consensus although experiments demonstrated that the generation of the collective behavior and the nature of the task, but also to a certain extent, illustrates the network structure will affect the behavior of the individual.

\section{Review and evaluation}

Relevant researches on social influence mainly focus on dynamics of social individual attributes and behaviors on static networks, such as Fowler's and Christakis's work. Some combination of network structure change of research are limited to the different structure of the static network or a particular point of the network structure, but the reality of social networks are evolve over time, the evolution of the network will affect the role of social influence is also worthy of further discussion.

In conclusion, individual interaction in collaborative networks is highly spontaneous and homophily inclined interaction mechanisms play a significant role in the interaction between individuals. At the same time, the change of network structure will form different degrees of social contagion, which will affect the change of individual attributes and behaviors. Therefore, the individual behavior and network structure in the collaborative network influence each other and evolve together.Schelling (1971) on a regular grid (to a certain extent, can be regarded as a rule network) a racial segmentation model is put forward, the earlier the race similar tendency in the analysis of the relationship between the neighbors will lead to social network by random state evolution for the division.Holme and Newman (2006) combined homophily with two social mechanisms that influence homophily and controlled each mechanism through the probability parameter values and mainly investigated the phase change process of community size and network structure. Kimura and Hayakawa (2008) added the heterophily mechanism into the Holme \& Newman model, and analyzed and concluded that the network would present a "small world" 
structure within a certain range of parameters. In addition, many researches have also conducted in-depth discussions on homophily and social network evolution of other social attributes. For example, Centola et al. (2007) analyzed the relationship between cultural similarity and social network structure[42]Grabowski and Kosi ń ski (2006) for cultural diversity and to explore the evolution of the network structure of common, Gracia - La Zaro Axelrod - Schelling model is put forward, and Lewis et al. (2011) for the online network homophily and the analysis of social contagion, etc.

Homophily's description of social behavior essentially includes two aspects, namely, the tendency of individuals to interact with other similar individuals and their tolerance of social attribute differences. Some existing related research work belongs to the former, such as Holme \& Newman model and Kimura \& Hayakawa model.In reality the interaction of the individual similarity judgment to interacting objects are always keep in a certain range, which means the individual attribute differences (social distance) in the range of acceptable level, social interaction can be formed.

Social contagion need individual behavior has the characteristics of socialization, namely the individual formed in the process of interaction of each other's mutual influence, the scope of this kind of influence is not unlimited, however, tend to be individual limits of the structure of social relations, which means that the structure of social networks for social impact formation and spread of have a certain effect, existing studies focus on static network structure under the social impact of related issues, as well as to show the relationship between different structure for the influence of the different role; The changes of network structure and interaction behavior are often interdependent. The dynamic characteristics of network structure need to be taken into account when considering social influence and interaction behavior. In addition, homophily and social contagion are intricately intertwined, and the effects of these two social mechanisms on interaction behavior and network structure need to be further distinguished.

Based on the above analysis, social collaboration network is a communication technology development and comes up with in the informal organization, compared with the formal organization, the behavior of the individual has a strong autonomy and the corresponding network structure also has a strong variability, the organization form of the loose, variable, however, tend to show the powerful ability to solve complex problems, so how to play this kind of informal organization to maximum efficiency, in turn, to solve the complex problems in social production, social collaboration network evolution mechanism is called for, and have a thorough understanding of relevant dynamics process, although the existing research work could be involved.

\section{References:}

[1] Josej.Ramasco,Dorogovtsev s. N., and pastor-satorras Romualdo. Self-organization of collaboration networks. Physical review E, 2004(70): 036106

[2] Moody J.The structure of a social science collaboration network: Disciplinary cohesion from 1963 to 1999. The American sociological review, 2004 (69): 213-238

[3] Newman M.E.J.Clustering and preferential attachment in growing networks. Physical Review E, 2001(64): 025102

[4] Schilling Melissa a. and Phelps Corey c. Interfirm collaboration networks: The impact of large scale network structure on firm innovation. Management Science, 2007(53): 1113-1126

[5] Powell Walter w., Koput Kenneth w. and smith-doerr Laurel. Interorganizational collaboration and the locus of innovation:

[6] Ahuja Gautam. Collaboration networks, structural holes, and innovation: A longitudinal study. Administrative science quarterly, 2000(45): 425-455 
[7] Newman M.E.J.. The structure of scientific collaboration networks. Of The National Academy of Sciences, 2001(98): 404-409

[8] camarinha-matos Luis m. and Afsarmanesh hamideh. Collaborative networks: a new scientific discipline. Journal of Intelligent Manufacturing, 2005(16): 439-452

[9] Gross Thilo and Blasius Bernd. Adaptive coevolutionary networks: a review. Journal of the Royal Society Interface, 2008(5): 259-271

[10] Centola D..An experimental study of homophily in the adoption of health behavior. Science, 2011(334): 1269-1272

[11] Christakis Nicholas a.and Fowler James h.Connected: The surprised power of our social networks and how they shape our lives. Hachette Digital Inc., 2009

[12] Clifford p. and Sudbury A.A model for spatial conflict. Biometrika, 1973(60), 581-588

[13] sznajd-weron k.and Sznajd j.Evolution in community closed. International Journal of Modern Physics C, 2000(11): 1157-1165

[14] Deffuant g., Neau d., Amblard f., and Weisbuch, G. Mixing beliefs among Interacting agents. Advances in Complex Systems, 2000 (3): 87-98.

[15] Galam s. Real space renormalization group and neighbor paradox of majority rule voting. Physica A, 2000(285): 66-76

[16] he R.The dissemination of culture. A model with local convergence and global polarization. Journal of conflict resolution, 1997, (41): 203-226

[17] Centola Damon, gonzalez-avella Juan Carlos, Eguiluz Victor $m$. and San Miguel maxi.homophily, cultural drift, and the co-evolution of cultural groups, Journal of Conflict Resolution, 2007(51) 